\title{
Obtaining molybdenum-copper composite for effective thermal control in electronic systems
}

\author{
Alexander Sivkov ${ }^{1}$, Yuliya Shanenkova ${ }^{1}$, and Yuliya Polovinkina ${ }^{1, *}$ \\ ${ }^{1}$ National Research Tomsk Polytechnic University, 634050 Tomsk, Russia
}

\begin{abstract}
In the article, the molybdenum and copper powders, obtained by electric explosion of wires and plasma-dynamic method, respectively, were sintered by spark plasma sintering technology, in order to obtain bulk sample based on them. X-ray diffractometry and scanning electron microscopy were carried out to study the chemical composition and structure of the resulting ceramics. XRD analysis showed that the product consisted of the following main phases such as molybdenum of a cubic crystalline structure (space group Im3m, No. 229) and copper of cubic system (space group Fm-3m, No. 225). The results of scanning electron microscopy indicated that the sintered sample had heterogeneous structure with the presence of small pores. Nonetheless, the mechanical properties such as hardness (Vickers hardness of $292 \mathrm{HV}$ ) and thermal conductivity $\left(140.53 \mathrm{~W} / \mathrm{m} \cdot{ }^{\circ} \mathrm{C}\right)$ are good enough that allows considering the proposed synthesizing methods as well as sintering technology as promising for creating the $\mathrm{Cu}-\mathrm{Mo}$ ceramics with high-mechanical properties.
\end{abstract}

\section{Introduction}

Increasing the energy density is one of the biggest problems for thermal regulation in small electronic devices. Indeed, the insufficient cooling negatively affects the reliability and efficiency of semiconductor components $[1,2]$. According to statistical data, more than half of the electronic components failure cases are explained with the increased temperature.

Electronic components are usually installed on substrates or plates, which act as the heat removal elements and provide an effective thermal control [3-5]. However, not all materials have suitable properties for reliable heat dissipation. In such substrates, the thermal conductivity and thermal expansion characteristics of the semiconductor must be matched with each other in the optimal way. The better the correlation between the properties, the better the semiconductor module withstands soldering during the production and temperature cycles in operation mode. In this paper, the bulk ceramics sample based on molybdenum and copper powders was sintered using the spark plasma sintering technology. Unlike the existing methods $[6,7]$, this technology makes it possible to obtain bulk samples based on powders in a short period of time that allows preserving the finegrained structure of the material and its unique properties. It is assumed that the molybdenum low thermal expansion and the copper excellent thermal conductivity will

\footnotetext{
* Corresponding author: xyulyashax@mail.ru
} 
provide substrates that can reliably dissipate heat in electrical components and help cool IGBT-modules, RF-modules, LED crystals and other products [8,9]. Also in this paper, a unique method for obtaining ultrafine copper powders based on using coaxial magnetoplasma accelerator is shown [10-12]. In comparison with other methods for obtaining ultrafine powders $[13,14]$, the discussed technique is more simple (working time less than $1 \mathrm{~ms}$ ) and doesn't require preliminary preparation of the main precursors.

\section{Materials and experimental procedures}

In order to obtain the bulk samples, the precursors in the form of ultrafine powders were used. Molybdenum was obtained using the method of electric explosion of wires (commercial powder), as well as copper was synthesized by plasmadynamic method. The unique installation based on the coaxial magnetoplasma accelerator with copper electrodes, which was used to obtain copper powder, is shown in figure 1.

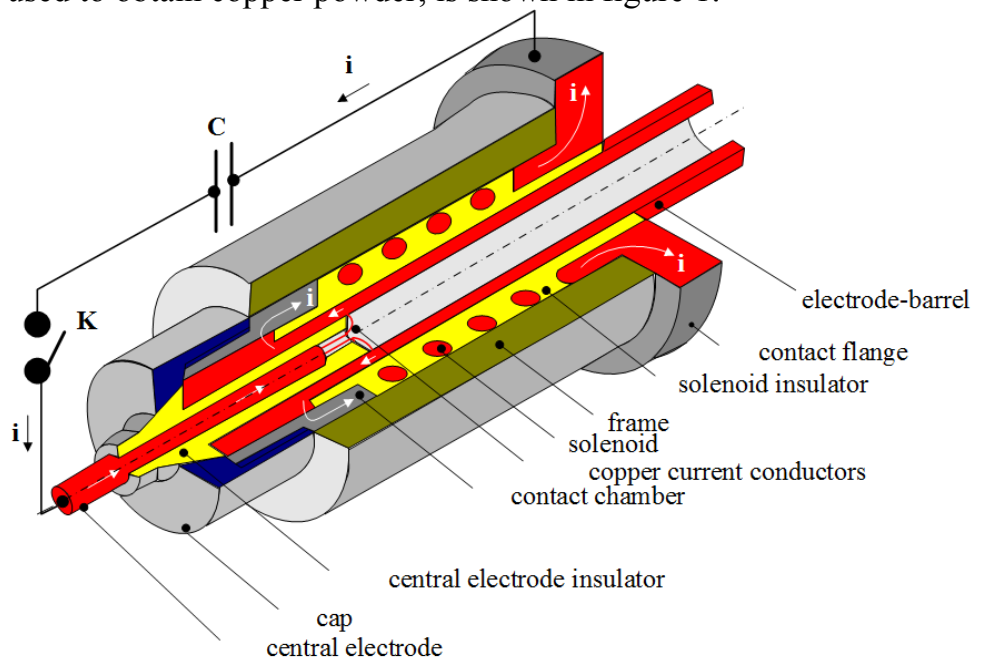

Fig. 1. Sketch-map and elements of coaxial magnetoplasma accelerator.

This device is based on the classical Z-pinch accelerator placed in an external induction system. The power supply of the accelerator is provided by a capacitive energy storage device with the following operating parameters: charge voltage up to $U=5.0 \mathrm{kV}$ and capacitance of capacitor banks up to $\mathrm{C}=28.8 \mathrm{mF}$. The Z-pinch accelerator consists of parts such as a central non-magnetic metal electrode with a copper insert at the end, a copper electrode-barrel, copper current conductors, a central electrode insulator, and power elements, holding this assembly. Z-pinch accelerator is placed in an external induction field, which is necessary to equalize the electroerosion wear along the length of the electrode-barrel.

After closing the power keys $(\mathrm{K})$, the capacitive energy storage device (C) discharges, therefore, the current increases immediately in the circuit; when it reaches to certain critical value, an electric explosion of the copper conductors takes place with the formation of an arc discharge. Due to the magnetic field the formed arc discharge of the solenoid is pulled along the electrode barrel. When the discharge propagates, the plasma jet interacts with the accelerating channel walls, and thus the copper production is performed due to electroerosion wear. Then the plasma escapes from the accelerating channel, getting into the chamber-reactor, where plasma-chemical reaction is implemented. The synthesized product settles down to the wall of the chamber. After one hour, the chamber is opened and 
the product is collected. It is worth nothing that the chamber-reactor was preliminary evacuated and filled with inert gas (argon) to prevent the saturation of the resulting material by oxygen (to avoid product oxidation).

Using a mix of such-obtained powders (copper and molybdenum) a bulk sample was obtained using a spark plasma sintering (Advanced Technologies 10-4 SPS). Cu and Mo powders were filled into the graphite molds (maximal pressure $\mathrm{P}_{\max }=60 \mathrm{MPa}$, maximal temperature $\mathrm{T}=2200{ }^{\circ} \mathrm{C}$ ) in the following rations of $30 \mathrm{wt} \%$ to $70 \mathrm{wt} \%$, respectively. The sintering process parameters were the following: the sintering atmosphere was vacuum, heating rate was $100{ }^{\circ} \mathrm{C} / \mathrm{min}$, , cooling rate was $500{ }^{\circ} \mathrm{C} / \mathrm{min}$, maximum heating temperature was regulated by the current flowing through the sample. Figure 2 shows the curves of temperature, sintering pressure and material density changes.

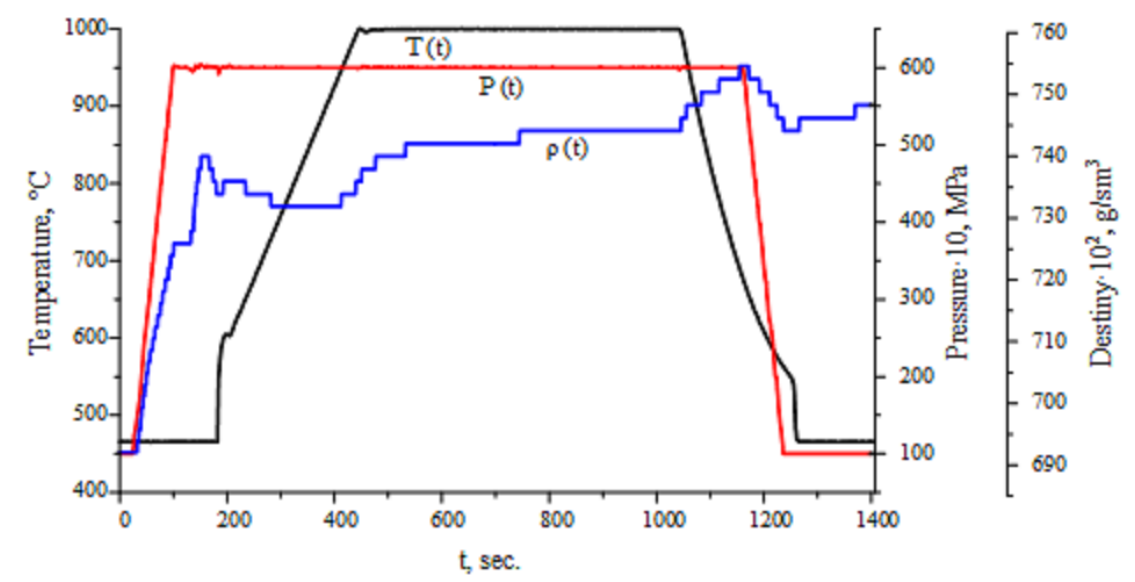

Fig. 2. Graph of temperature, sintering pressure and material density changes.

XRD. X-ray diffractometry analysis was carried out using a Shimadzu XRD-7000S diffractometer with $\mathrm{Cu}-\mathrm{K} \alpha$ radiation equipped with the counter monochromator. The phasestructural analysis has been made using the database PDF2 and the program software PowderCell 2.4.

SEM. Scanning electron microscopy of the sintered ceramics was carried out using a Hitachi TM-3000 scanning electron microscope.

Thermal conductivity. Thermal conductivity measurement was studied using a Discover Flash DLF-1200 thermal diffusivity analyzer.

Hardness. The hardness of the bulk sample was determined by a Vickers hardness tester Galileo Durometer Isoscan HV2 with a sample load of $0.5 \mathrm{kgf}$.

\section{Results and discussion}

As a result of sintering process, the dark grey ceramics sample was obtained. Its geometrical sizes were found (the diameter of $12.7 \mathrm{~mm}$, the height of $4 \mathrm{~mm}$ ) in order to calculate the resulting density, which was found to be equal to $7.48 \mathrm{~g} / \mathrm{sm}^{3}$. Figure 3 shows the SEM-image of the sintered $\mathrm{Cu}-\mathrm{Mo}$ ceramics sample. It can be seen that the material is very heterogeneous in structure. There are both nanoparticles and large agglomerations of $20 \mu \mathrm{m}$ and larger. This unsatisfied structure and a relatively low sample density $(\rho=76 \%)$, allows us to conclude that the solid sample obtained under such conditions is not of a sufficient quality because of a significant number of pores, the presence of which can be easily explained by the low sintering temperature. It can be seen that the molybdenum 
particles are embedded in the copper matrix. The SEM-image allows also finding the presence of big white inclusions, the nature of which was not understood until the carrying out XRD analysis.

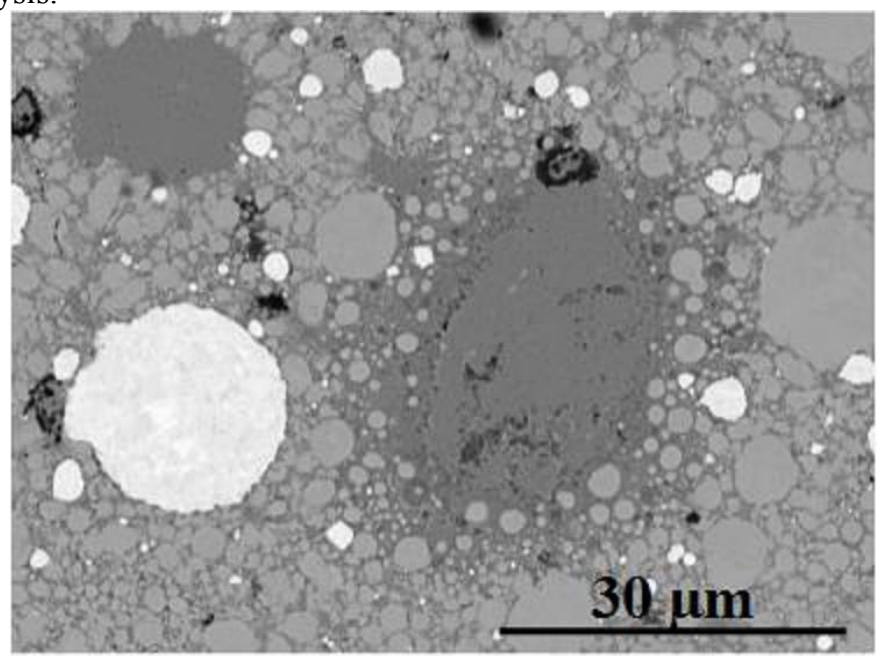

Fig. 3. SEM-image of sample.

Figure 4 shows X-ray diffraction pattern of the synthesized product. This figure shows that the product consists of the following main phases such as molybdenum of cubic system (space group Im-3m, No. 229) and the copper of a cubic crystalline structure (space group Fm-3m, No. 229). There are also a number of low-intensity peaks in the $2 \theta$ range from 30 to 40 degrees. The identification of these peaks in the PowderCell 2.4 software allowed attributing these peaks to the phase of tungsten, which can be included in the ceramics sample with the molybdenum powder. The process features electric explosion of wires cannot prevent from obtaining tungsten particles, that is why some of them are found on $\mathrm{XRD}$ as well as SEM data. Nonetheless, the dominant phases are $\mathrm{Mo}$ and $\mathrm{Cu}$ and their content in the resulting ceramics are $70.39 \%$ and $29.61 \%$, respectively.

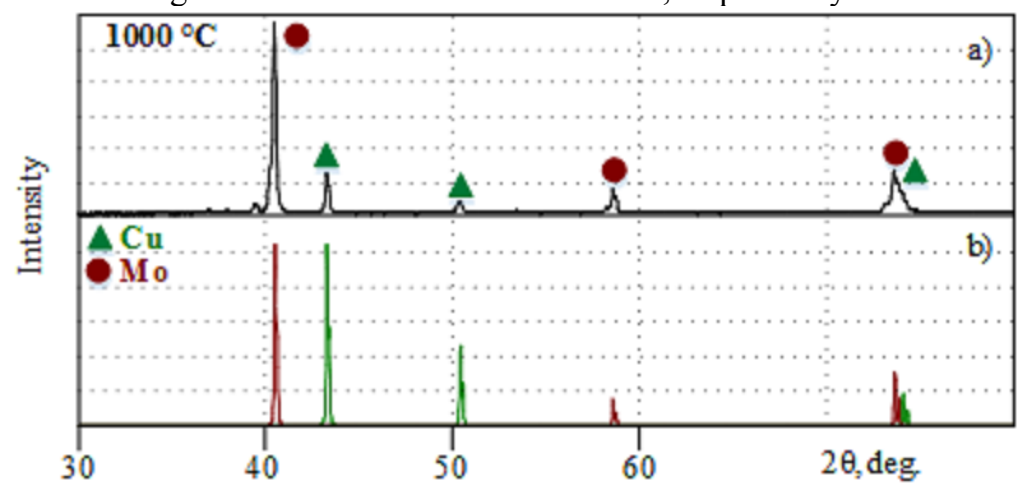

Fig. 4. XRD patterns of: a) sintered product, b) reference phases.

In order to estimate the possibility of using such ceramics in heat-conducting plates, such mechanical properties as thermal conductivity and hardness were studied. The measurements of thermal conductivity, which were carried out at room temperature, showed that the maximum thermal conductivity of the material was $140.53 \mathrm{~W} / \mathrm{m} \cdot{ }^{\circ} \mathrm{C}$. This parameter can be considered as good enough in comparison with known data [15], however it can be improved further by increasing the sintering temperature in order to melt all 
copper, which was found in the structure in the form of large aggregates (fig. 3). The presence of pores between $\mathrm{Cu}$ aggregates and Mo particles affects the Vickers hardness value which was equal to $292 \mathrm{HV}$. This value is much greater than for pure molybdenum $(147 \mathrm{HV})$, nonetheless it can also be improved with increasing the density of ceramics.

\section{Conclusion}

This paper showed the possibility of obtaining a bulk ceramics material based on ultrafine powders of molybdenum and copper. The methods for obtaining $\mathrm{Cu}$ powders and a ceramics sample were considered in detail. The presence of $\mathrm{Mo}$ and $\mathrm{Cu}$ phases in the final sample was confirmed by the results of X-ray diffraction analysis. The microstructure of the sample was also studied. To sum up, it is necessary to increase the sintering temperature to obtain the sample with the higher density of the material. This fact should have a positive effect on the material heat conductivity, which can increase with decreasing the pore number. Nonetheless, the not too high density does not affect the hardness of ceramics sample, which was found to be equal to $292 \mathrm{HV}$ that is much higher than for pure molybdenum.

\section{References}

1. G.V. Kuznetsov, D.V. Feoktistov, E.G. Orlova, K.A. Batishcheva, Colloid J. 78, 335 (2016)

2. D. Feoktistov, E. Orlova, A. Islamova, EPJ, 110, 01018 (2016)

3. N.Y.A. Shammas, Microelectron. Reliab. 43, 519 (2003)

4. A.B. Lostetter, F. Barlow, A. Elshabini, Microelectron. Reliab. 40, 365 (2000)

5. R. Hocine, A. Boudghene Stambouli, A. Saidane, Microelectron. Reliab. 65, 293 (2003)

6. A.G. Karengin, A.A. Karengin, I.Y. Novoselov, N.V. Tundeshev, Adv. Mater. Research, 1040, 433 (2014)

7. A.A. Karengin, A.G. Karengin, V.A. Vlasov, Russian Physics J. 58, 730 (2015)

8. D. Srinivasan, P.R. Subramanian, Mater. Sci. Eng. 459, 145 (2007)

9. B. Wang, S.H. Liang, J.T. Zou, X.H. Yang, P. Xiao, Rare Metal Mater. 40, 1621 (2011)

10. A. Sivkov, A. Ivashutenko, Y. Shanenkova, I. Shanenkov, J. Adv. Powder Techn. 27, 1506 (2016)

11. A.A. Sivkov, A.Y Pak, D.S. Nikitin, I.A. Rakhmatullin, I.I Shanenkov, Nanotech. in Russia, 8, 489 (2013)

12. A. Sivkov, A. Saygash, J. Kolganova, I. Shanenkov, IOP Conference Series: Mater. Sci. Engin. 66, 012048 (2014)

13. E.B. Kasharov, N.N. Nikitenkov, A.N. Sutygina, M.S. Syrtanov, O.V. Vilkhivskaya, etc., J. Surface Technol. 308, 2 (2016)

14. V.N. Kudiiarov, E.B. Kasharov, M.S. Syrtanov, I.S. Yugova, IOP Confer. Series: Mater. Science Engin. 110, 012071 (2016)

15. D. Wang, B. Yin, A. Sun, X. Li, C. Qi, B. Duan, J. of Alloys Compounds, 674, 347 (2016) 